\title{
Jointly Learning Non-negative Projection and Dictionary with Discriminative Graph Constraints for Classification
}

\author{
Weiyang Liu ${ }^{12}$ \\ wyliu@pku.edu.cn \\ Zhiding $\mathrm{Yu}^{3}$ \\ yzhiding@andrew.cmu.edu \\ Yandong Wen ${ }^{3}$ \\ yandongw@andrew.cmu.edu \\ Rongmei Lin ${ }^{4}$ \\ rongmei.lin@emory.edu \\ Meng Yang*1 \\ yang.meng@szu.edu.cn
}
${ }^{1}$ College of Computer Science \& Software Engineering, Shenzhen University, China

${ }^{2}$ School of ECE, Peking University, China

${ }^{3}$ Dept. of ECE, Carnegie Mellon University, USA
${ }^{4}$ Dept. of Math \& Computer Science, Emory University, USA

Different from the conventional wide variety of discriminative Dictionary learning (DL) literatures, our work casts an alternative view on this problem. One major purpose of this paper is to jointly learn a feature projection that improves DL. Instead of keep exploiting additional discrimination from the dictionary representation, we consider optimizing the input feature to further improve the learned dictionary. We believe such process can considerably influence the quality of learned dictionary, while a better learned dictionary may directly improve subsequent classification performance.

Given that mid-level object parts are often discriminative for classification, we aim to learn a feature projection that mines these discriminative patterns. It is well-known that nonnegative matrix factorization (NMF) [1] can learn similar part-like components. In the light of NMF and projective NMF (PNMF) [2], we consider the projective self-representation ( $\mathrm{P}$ SR) model where the set of training samples $\boldsymbol{Y}$ is approximately factorized as: $\boldsymbol{Y} \approx \boldsymbol{M} \boldsymbol{P} \boldsymbol{Y}$. The model jointly learns both the intermediate basis matrix $\boldsymbol{M}$ and the projection matrix $\boldsymbol{P}$ with non-negativity such that the additive (nonsubtractive) combinations leads to learned projected features $\boldsymbol{P} \boldsymbol{Y}$ accentuating spatial object parts. In the paper, we propose a novel NMF-like feature projection learning framework on top of the PSR model to simultaneously incorporate label information with discriminative graph constraints. One shall see, our proposed framework can be viewed as a tradeoff between NMF and feature learning [4].

The dictionary representation is further discriminatively learned given the projected input features. An overview of the joint non-

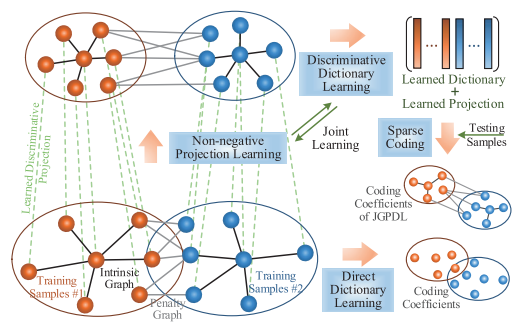

Figure 1. An illustration of JNPDL.

negative projection and dictionary learning (JNPDL) framework is illustrated in Fig. 1. The construction of discriminative graph constraints in both non-negative projection and dictionary learning follows the graph embedding framework [3]. While the inputs of graph constraints are essentially the same, they form different regularization terms for the convenience of optimization. Finally, a discriminative reconstruction constraint is also adopted so that coding coefficients will only well represent samples from their own classes but poorly represent samples from other classes. We test JNPDL in both image classification and image set classification with comprehensive evaluations, showing the excellent performance of JNPDL.

[1] Daniel D Lee and H Sebastian Seung. Learning the parts of objects by non-negative matrix factorization. Nature, 401(6755):788-791, 1999.

[2] Xiaobai Liu, Shuicheng Yan, and Hai Jin. Projective nonnegative graph embedding. IEEE T. IP , 19(5):1126-1137, 2010.

[3] Shuicheng Yan, Dong Xu, Benyu Zhang, Hong-Jiang Zhang, Qiang Yang, and Stephen Lin. Graph embedding and extensions: a general framework for dimensionality reduction. IEEE T. PAMI, 29(1):40-51, 2007.

[4] Will Zou, Shenghuo Zhu, Kai Yu, and Andrew Y Ng. Deep learning of invariant features via simulated fixations in video. In NIPS, 2012. 